DE DE GRUYTER

OPEN

BULGARIAN ACADEMY OF SCIENCES

CYBERNETICS AND INFORMATION TECHNOLOGIES • Volume 16, No 2

Sofia • $2016 \quad$ Print ISSN: 1311-9702; Online ISSN: 1314-4081

DOI: $10.1515 /$ cait-2016-0029

\title{
Advanced Control of the Wood Thermal Treatment Processing
}

\author{
Mincho Hadjiski ${ }^{1,2}$, Nencho Deliiski ${ }^{3}$ \\ ${ }^{1}$ Institute of Information and Communication Technologies, BAS, 1113 Sofia, Bulgaria \\ ${ }^{2}$ Chemical-Technology and Metallurgy University, 1756 Sofia, Bulgaria \\ ${ }^{3}$ University of Forestry, 1756 Sofia, Bulgaria \\ Emails: hadjiski@uctm.edu deliiski@netbg.com
}

\begin{abstract}
An advanced control system is considered for wood Thermal Treatment Processing (TTP) in order to cope with the scarcity of on-line measurements as well as cope with a variety of unpredictable operational conditions arising due to disturbances and large uncertainties. The architecture of the proposed control system includes a number of Building Blocks (BB), functionally based on well-established algorithms, so that that the design and operation of the system use mainly parameterisation via simulation, optimization and coordination. In the created $B B$ a set of advances are incorporated: first principle modelling of WEP, inference control, Run-to-Run optimization, Case-Based Reasoning (CBR). Some results of real applications in SMEs are presented.
\end{abstract}

Keywords: Advanced process control, Case-Based Reasoning (CBR), inference control, Thermal Treatment Processing (TTP), Run-to-Run optimization.

\section{Introduction}

Wood continues to be traditionally in many cases a preferable construction material for designers in the furniture industry and houses fitting-up. But for the wood conversion from raw substance into an industrial product, some kind of thermal/hydrothermal processing is obligatory. Now the wood Thermal Treatment Processing (TTP) remains the most established technology in use [9, 21, 24, 25]. The TTP is already deeply studied during the years as purely a technological problem $[24,25]$. In the last decades some dynamic aspects of WEP, and especially the wood thermal treatment, have been considered, using the first principle mathematical models presented by partial differential equations, a basis for computer model creation $[7,8,9,11,13]$.

As TTP is a considerably energy and time consuming process [8, 11, 24, 25], a problem arises for cost and quality effectiveness. Unfortunately, low attention has 
been paid to the investigation and improvement of the corresponding control system of TTP. To meet both strong requirements for plasticity and colouring from the side of the designers and the competition reasons as well as a new generation of advanced control systems is needed. Some results have been reported $[13,14,15]$ but they are addressed mainly toward the guarantee only of the plasticity of the wood needed for the subsequent mechanical processing.

The problem of achieving a desired change of the natural colour of the wood and of its structure during the TTP became very important during the last years according to market requirements. The results of the technological investigations $[6,12,21]$ show that the main problems for satisfactory control are the space distribution of the diffusion process of colouring and restructuring as well as the impossibility of on-line wood colour measurement during the TTP tracking. Fortunately, some new opportunities have appeared lately, based on new developed sensor systems for colour measurement [12,21].

Unfortunately, the only discrete colour information is available at the end of each run, because the TTP is a periodical (batch) process. In this paper the functional possibilities of the earlier proposed control system for TTP $[13,14,15]$ are enhanced with emphasis both on the plasticizing and ennoblement (colouring) of the wood via incorporating a new set of Building Blocks (BB) into the control scheme: direct colour measurement at the end of each run [12, 21], adaptation of tuning parameters of the accepted model based inference control scheme [18, 19], Run-to-Run (RtoR) optimization and re-parameterization [3, 28], operational knowledge inclusion in the form of Case-Based Reasoning (CBR) [4, 6, 16, 17, 27].

\section{Thermal treatment processing}

The Thermal Treatment Processing (TTP) is a periodical process of heating and subsequent cooling of the wood materials. Depending on the needed temperature of the heating medium, $t_{\mathrm{m}}$, it is carried out in autoclaves with $t_{\mathrm{m}}=100$ up to $140{ }^{\circ} \mathrm{C}$ ) or in pits with $t_{\mathrm{m}}=100$ up to $140^{\circ} \mathrm{C}$ (Fig. 1). The only temperature of the heating fluid (steam or hot water), $t_{\mathrm{m}}$, and their flow rates $G$ could be measured on-line. The main passing processes - heat exchange and diffusion are strongly mutually connected due to common local temperature $T(x, y, z)$ and average mass temperature of the subjected to TTP wood, $\bar{T}$ (in K).

The generalized scheme of plasticizing and ennoblement of the wood is presented on Fig. 2, where $\mu$ and $v$ mean the relative degrees of the wood plasticity and wood coloring respectively.
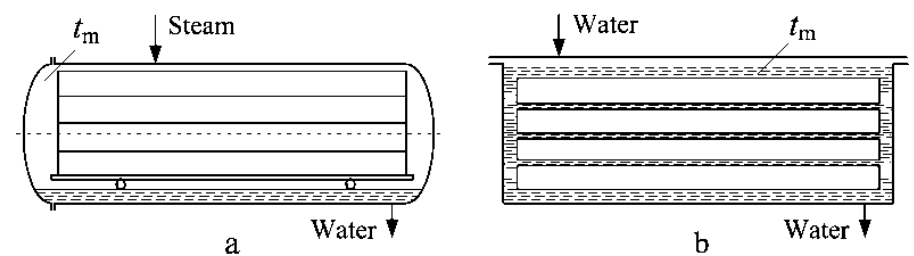

Fig. 1. Autoclave (a) and pit (b) for thermal treatment of wood materials 


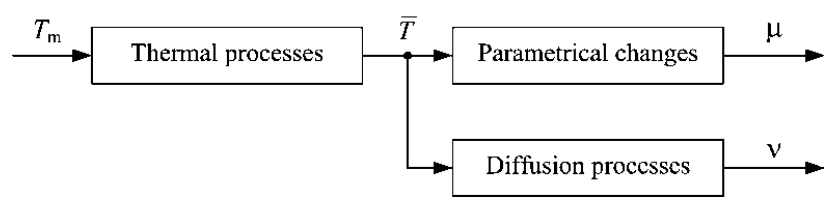

Fig. 2. Main processes in the ennoblement of the wood by TTP

A typical time profile of the temperature changes during the run $\left(\tau_{\mathrm{p}}=12 \mathrm{~h}\right)$ in different points of the wood space using computer simulation [9], are shown on Fig. 3 for a subjected to autoclave steaming beech $\log$ with diameter $D=0.4 \mathrm{~m}$ (i.e., radius $R=0.2 \mathrm{~m}$ ), length $L=0.8 \mathrm{~m}$, and initial temperature $t_{0}=0{ }^{\circ} \mathrm{C}$.

The transient response in the dynamic channel $\bar{T}-\mu$ is relatively fast in comparison to the $\bar{T}-v$ channel due to slow diffusion, as shown on Fig. 4.

The TTP is organized by a sequence of batches and runs. Each batch contains a predefined volume of wood materials, set from the planning and scheduling scheme, sharing similar characteristics - wood specie, form and dimensions, average initial parameters: moisture content $u^{\circ}$, temperature $T^{\mathrm{o}}$, and basic density $\rho_{\mathrm{b}}$. The batch is divided into individual charges - "runs". Each run could be different in regards to the "main statistical" one due to the changes in the wood characteristics as well as the level of charging $L$. Thus run to run could differ considerably and this is where a lot of the uncertainties with regards to the thermal treatment processing of wood materials originate from.

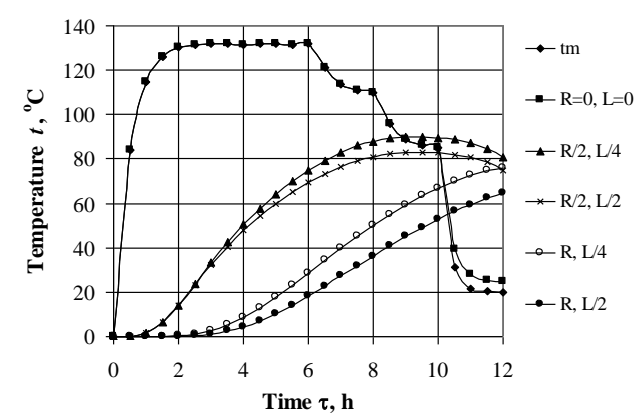

Fig. 3. Change in $t_{\mathrm{m}}$ and $t$ in five characteristic points of the longitudinal section of beech log with $D=0.4 \mathrm{~m}, L=0.8 \mathrm{~m}$, and $t_{0}=0{ }^{\circ} \mathrm{C}$ during autoclave steaming and subsequent conditioning at $20^{\circ} \mathrm{C}$

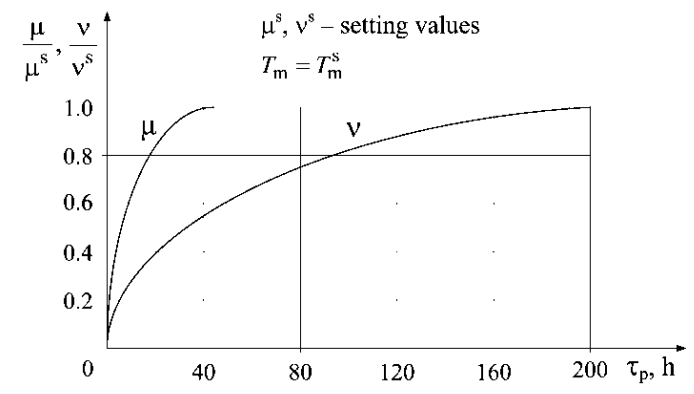

Fig. 4. The dynamic processes of plasticization $(\mu)$ and coloring $(v)$ 
On the other hand, the TTP is subjected to a number of disturbances - part of them are due to the operational decisions on the supervisory level (e.g., correction of the reference values of the terminal time $\tau_{\mathrm{p}}$ ), another part are caused by the heating fluid parameters' changes (pressure, enthalpy) and from variations of the environmental conditions.

\section{Measurement and color estimation}

In industrial scale production the wood color could be measured only at the end of each run. Thus the only iterative algorithms using discrete information could be used in order to improve the control system performance in order to cope with uncertainty and disturbances.

One of the methods for objective quantifying of this wood optical property is expressing it in the coordinates of the CIE-L*a*b* colour space. A colour coordination system CIE1976 is based on the measurement of three parameters: lightness $\mathrm{L}^{*}$ from 100 for white to 0 for black, chromatic coordinate $a^{*}$ for determination of the shade between red and green colour and chromatic coordinate $b^{*}$ determining the shade between yellow and blue colour.

On Fig. 5 the colour of the wood, point A on the spherical plane of CIE colour space, is shown.

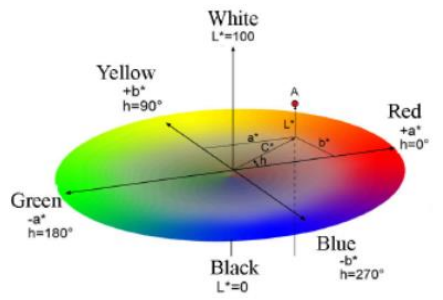

Fig. 5. The colour of the wood, determined by $L^{*}, a^{*}, b^{*}$ coordinates in the CIE colour space

The values of the colour space coordinates could be expressed in the form of $x=\bar{x} \pm u_{\mathrm{c}}$, where $\bar{x}$ are the average measured values of the colour coordinates and $u_{\mathrm{c}}$ is the combined standard measured uncertainty.

Variance of the determined coordinate values in the CIE-L*a*b* colour space of steamed and non-steamed details could be evaluated using the relative standard uncertainty $u_{\mathrm{c}}^{\text {rel }}$.

The values of the relative standard uncertainty for particular colour coordinates could be determined according to the following equation:

$$
u_{\mathrm{c}}^{\text {rel }}=\frac{u_{\mathrm{c}_{i}}}{\overline{x_{i}}},
$$

where $u_{\mathrm{c}}^{\text {rel }}$ is the relative standard uncertainty of the colour space coordinates, \%;

$u_{\mathrm{c}_{i}}-$ combined standard uncertainty of the colour space coordinates values of each of studied details; 
$x_{i}$ - average arithmetic value of the colour space coordinates;

$i$ - serial number of the studied details: $i=1, \ldots, N$.

Based on the differences of the separate colour parameters $\Delta L^{*}=L_{2}^{*}-L_{1}^{*}$, $\Delta a^{*}=a_{2}^{*}-a_{1}^{*}$, and $\Delta b=b_{2}^{*}-b_{1}^{*}$ the total colour deviation $\Delta E^{*}$ could be determined according to following equation of ISO 11 664-4:

$$
\Delta E^{*}=\sqrt{\left(L_{2}^{*}-L_{1}^{*}\right)^{2}+\left(a_{2}^{*}-a_{1}^{*}\right)^{2}+\left(b_{2}^{*}-b_{1}^{*}\right)^{2}},
$$

where $L_{1}^{*}, a_{1}^{*}, b_{1}^{*}$ are the colour space coordinates of the non-steamed beech details;

$L_{1}^{*}, a_{1}^{*}, b_{1}^{*}-$ the colour space coordinates of the steamed beech details.

\section{Problem statement}

This paper is a continuation of previous works [13, 14, 15] for recursive control design of controls system for TTP via progressive augmentation of the control structure adding new Building Blocks with the goal to improve the whole TTP performance.

\subsection{Starting structure}

1. The design process starts with an internal control system with PLC control, based on static optimization [11].

2. The next step is an open loop control using dynamic optimization of the mean mass temperature $\bar{T}$ of the run material, based on simplified suboptimal control algorithm [15].

3. Incorporation of an approximated model of the internal thermal process as inference control augmentation [13] with correction of the partial regime parameters [14].

The obtained control structure improves the TTP, but experimental results of the commissioned industrial systems show that a significant reserve still exists for the performance to be better. The main reason is that the achieved control system strongly depends on existing uncertainties in each run due to the changes of the biological properties $\xi$ of the wood material, of its initial temperature $T^{0}$ and moisture content $u^{\mathrm{o}}$.

As the main mass temperature of the wood material $\bar{T}(k)$ is immeasurable, the inference control into the adopted structure could give as a result a highly sub-optimal control in cases when strong deviations between the real parameters $\xi$, $u^{\circ}$, and $T^{\mathrm{D}}$ for the particular run and respective accepted averaged values for the current charge occur. Hence handling uncertainties becomes an important issue, especially in our case when the terminal constrains are hardly related to the quality and productivity of the TTP.

4.2. The main goals for the improvement of the control system

The main goals for the improvement of the control system are, as follows:

- To enhance control functions from only plasticization of the wood materials toward both their plasticization and ennoblement (mainly coloring). 
- To attenuate the impact of the model parameters and uncertainties on the degree of sub-optimality of the hating medium $T_{\mathrm{m}}$ tracking and the terminal constraints satisfaction.

- To use different measurements of the wood color at the end of each run in order to correct both mathematical models and initial settings for suboptimal control.

- To achieve an improvement of the whole TTP in the next aspects:

- quality growing of the ennoblement;

- flexibility arising in respect to Management Acquisition System (MAS) requirement satisfying and all possible operational regimes covering;

- operational simplicity remaining (easy to understand, transparency, low psychological load;

- cost orientation using general purpose devices (controllers, industrial computers) and established algorithms.

- To coordinate rigorous first principle off-line models, on-line measurements, and knowledge blocks.

\subsection{Peculiarities of the TTP-control problem}

The TTP has a number of peculiarities, which impact the choice of relevant approaches accepted in the design in this work, as follows:

- The process is periodical. The strong results of investigations received in the chemical industry about the control of "batch-to-batch" ("run-to-run") processes $[3,28]$ could be a solid base for the incorporation of color measurement at the end of each run;

- The process is with space dependence on parameters. Thus only scarce direct measurements are available for the state of wood temperature $T(x, y, z, \tau)$ and $v(x, y, z, \tau)$ as well as for their derivatives;

- The process of plasticization and ennoblement are nonlinear. They depend on changeable from run to run parameters

$$
P=\left\langle P_{1}, P_{2}\right\rangle,
$$

where $P_{1}=\left\langle\rho_{\mathrm{b}}, \xi\right\rangle$ are parameters specifying the wood specie and the peculiarities of the wood: $\rho_{\mathrm{b}}$ is the basic density of the wood (equal to green mass divided by green volume); $\xi$ is a measure of the microstructure of the wood; $P_{2}=\left\langle\bar{D}, L^{\mathrm{o}}, u^{\mathrm{o}}, T^{\mathrm{o}}\right\rangle$ are parameters different for each run: $\bar{D}$ is average space measure (dimension) for treated into the run wood materials; $L^{\mathrm{o}}$ is the relative load of the run:

$$
L^{\mathrm{o}}=\frac{L}{L_{\max }}
$$

where $L$ and $L_{\max }$ are the real and the maximum possible load respectively in the given $R_{i, j}$ run (i.e., in $j$-run from $i$-charge of the autoclave or pit).

The parameters $u^{\mathrm{o}}$ and $T^{\mathrm{o}}$ are the initial moisture content and temperature of the wood materials, subjected to TTP in $(i, j)$-run.

For each $i$-carge $C_{i}$ some averaged values of the components of $P_{i}$ could be defined: 


$$
\bar{P}_{i}=\left\langle P_{i 1}, P_{i 2}\right\rangle,
$$

but for the separate runs $R_{i, j}$, belonging to the charge $C_{i}$, the individual parameters $P_{i, j, 1}, P_{i, j, 2}$ are different:

$$
P_{i, j, k}=\bar{P}_{i, j, k}+\delta P_{i, j, k} \quad \text { if } \quad k=1,2 .
$$

The variations $\delta P_{i, j, k}$ have stochastic nature and depend on non-predictable bioand technological reasons, connected with the growth, obtain, storage, and making up of $C_{i}$ charge and $R_{i, j}$-run.

The nonlinearity and parametric dependence are an origin of considerable uncertainty in TTP.

- The control system is subjected to number of constrains:

$T_{\mathrm{m}}^{\min }<T_{m}<T_{\mathrm{m}}^{\max }$ - heating medium temperature constrains;

$\frac{\partial T(x, y, z, k)}{\partial n}<\Gamma_{1}-$ gradient of the admissible temperature space of the wood material;

$\frac{\partial T(x, y, z, k)}{\partial \tau}<\Gamma_{2}-$ gradient of the admissible temperature during the time of TTP of the wood material;

$\left|v\left(x, y, z, \tau_{\mathrm{p}}\right)-v^{\mathrm{o}}\right|<\delta_{v}-$ admissible terminal color deviation for each point of the wood material;

$T_{\mu}^{\min }<\bar{T}\left(\tau_{\mathrm{p}}\right)<T_{\mu}^{\max }-$ admissible deviation of the terminal average mass temperature for the plasticization's process.

- The constraint values (thresholds) depend on a variety of circumstances and are usually defined experimentally in iterative way.

\section{Advanced control system of TTP}

The main peculiarities of the proposed advanced control system are presented below with a stress on Run-to-Run optimization of the model parameters.

\subsection{The design principles}

The next design principles have been leading in this work:

- To create a control structure on the basis of iterative adding of new functional Building Blocks, such as the back stepping principle of Kokotovich et al., given in [29], but addressed toward improving the level of performance, not only achieving of the asymptotic stability;

- To use mainly parametric optimization, not control algorithm synthesis;

- To attain a flexibility by changing structure or parameters of the control system depending on the real operational situation;

- To use well established Building Blocks and their algorithms with minor modification if possible; 
- To emphasize on co-ordination of variety of Building Blocks instead of trying to obtain unified nonlinear control algorithm;

- To find a relevant form of operational knowledge representation for control in cases of rare and unpredictable situations.

The scheme of the proposed TTP control system is presented on Fig. 6. It contains a number of Blocks, which will be described below in details.

\subsection{Supervisory control}

The supervisory control has the goal to generate the initial optimal (or suboptimal) values of the heating medium temperature $T_{\mathrm{m}}$ and of the heating time $\tau_{\mathrm{p}}$ for each technological cycle.

The objectives of the supervisory control could be, as follows:

- To guarantee a given capacity $\mathrm{J}_{1}$ :

- $J_{11}$ - to minimize the heat consumption;

$-J_{12}$ - to minimize the time of the operation;

$-J_{13}$ - to find the extreme value of the multi objective function

$$
J_{13}=\gamma J_{11}+(1-\gamma) J_{12} \rightarrow \text { extr. }
$$

- To maximize the productivity $\mathrm{J} 2$ :

$$
J_{2} \rightarrow \max \text {. }
$$

In generalized form the objective function could be presented in the following form:

$$
J=\left\langle J_{1}, J_{2}\right\rangle
$$

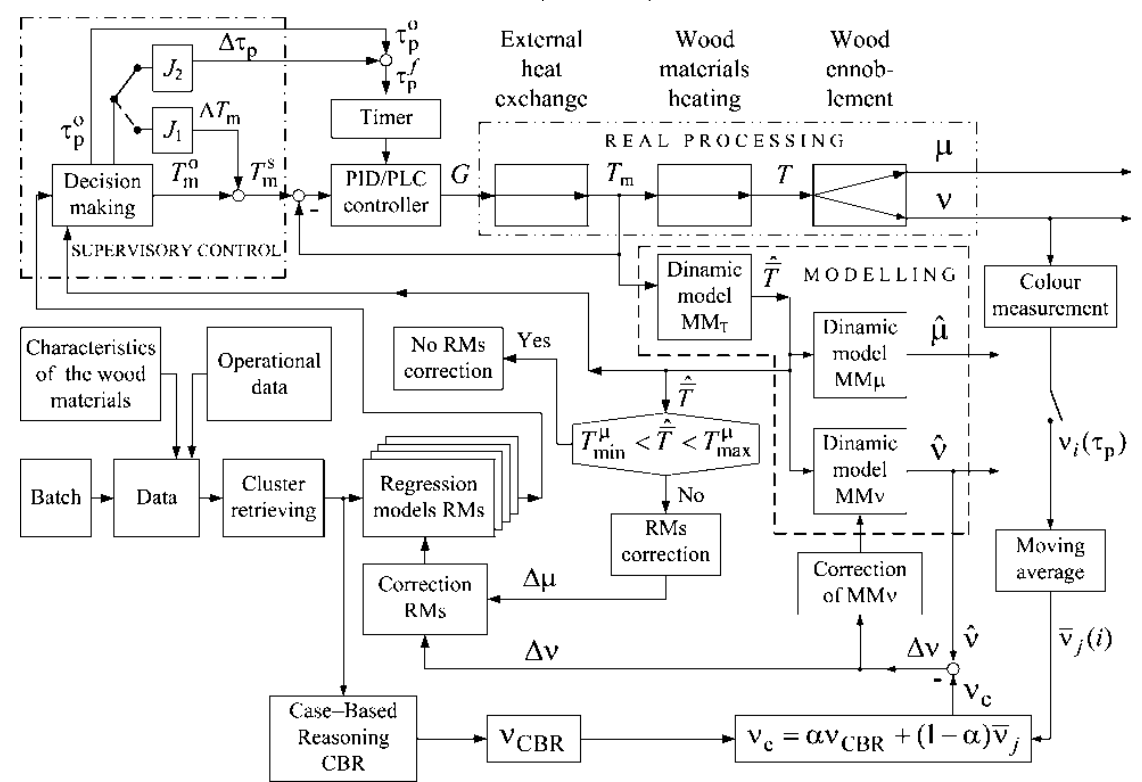

Fig. 6. Scheme of inference control of thermal treatment of wood materials, including CBR

The problem to be solved in $\mathrm{BB} 1$ is

$$
J\left(T_{\mathrm{m}}, \tau_{\mathrm{p}}\right)=\arg \min J\left(T_{\mathrm{m}}, \tau_{\mathrm{p}}\right),
$$


subjected to

$$
\begin{gathered}
T_{\mathrm{m}}<T_{\mathrm{m}}^{\max }, \\
G<G^{\max }, \\
\frac{\partial T(x, y, z, p)}{\partial n} \leq \Gamma_{1}, \\
\frac{\partial T(x, y, z, p)}{\partial \tau} \leq \Gamma_{2}, \\
T_{\mu}^{\min }(p)<\bar{T}^{f}<T_{\mu}^{\max }(p), \\
\left.\frac{\partial v(x, y, z, p)}{\partial n}\right|_{\tau_{\mathrm{p}}}<\Gamma_{3}, \\
\left|\bar{v}^{f}(p)-v^{\mathrm{o}}\right|<\Gamma_{4} .
\end{gathered}
$$

The constrains (10) and (11) guarantee flexibility of the TTP. Conditions (12) and (13) ensure the homogeneity of the temperature in the subjected to TTP wood material and at the time. Terminal constraints (14), (15), and (16) guarantee accomplishment of the requirements for the plasticity and for the coloring of the wood $[6,12,21,24,25]$. For decision making based on the optimization a necessary information is needed: the characteristics of the wood material in the current batch $\left(D, L, u^{\mathrm{o}}, T^{\mathrm{o}}, \rho_{\mathrm{b}}, \xi\right)$, operational data $\left(\tau_{\mathrm{p}}^{\mathrm{o}}, G^{\max }, v\right)$, current prices of heat, data from regression models $[13,14]$.

Depending on the business goal reformulation during the run some corrections of the control variable $\Delta T_{\mathrm{m}}$ and the terminal time $\Delta \tau_{\mathrm{p}}$ are possible.

\subsection{Basic control}

The basic control is realized by standard PID or PLC controller in order to track and stabilize the main control variable $T_{\mathrm{m}}$.

\subsection{Model based inference control of the wood average mass temperature $\bar{T}$}

The dynamic mathematical model $\mathrm{MM}_{\mathrm{T}}$ is derived using the first principle methods for nonlinear heat exchange $[9,11,13,15]$. A simplified model for the average mass temperature $\bar{T}(k)$ is received in the following discrete form:

$$
\bar{T}(k+1)=f(\bar{T}(k), \bar{P}, k)+g(T(k), \bar{P}) T_{\mathrm{m}}(k),
$$

where $\bar{P}$ is an approximate parameter vector and $k$ is discrete time.

The approximate estimates of the constraints (12), (13), and (14) are calculated using the full mathematical model $\mathrm{MM}_{\mathrm{T}}$ or according eq. (17). The accuracy of the $\hat{T}(x, y, z, k)$ and $\hat{\bar{T}}(k)$ strongly depends on the parametric vector $\bar{P}$ deviations:

$$
\Delta P=\|\bar{P}-P\| \text {. }
$$

As the suboptimal control of TTP is defined with respect to the approximate model (17) [13-15], the performance of this inference control system to a high degree 
depends on the uncertainty $\Delta P$, which could not be compensated by real feedback, not by its estimate $\hat{\bar{T}}$.

Nevertheless, incorporating the mathematical model $\mathrm{MM}_{\mathrm{T}}$ into the closed control system improves significantly its performance, especially in cases of relatively small $\Delta P$ deviations $[13,14]$.

\subsection{Color measurement}

The estimation of the color and the constraint (16) of the processed wood material could be carried out only at the end of each $j$-run of the current $i$-batch. Because the $i$-batch is formed in this way to use the wood material with similar characteristics, described with parametric vector $\bar{P}_{i}$, it is appropriate to use the estimate of the current color in $j$-run $v_{i}\left(\tau_{\mathrm{p}}\right)$ as Moving Average value (MA) from the previous measurements $j$ :

$$
v_{j}(i)=\frac{1}{l+1} \sum_{r=j-l-1}^{j} v_{\mathrm{r}}, i=1,2, \ldots, N_{\mathrm{c}}, j=1,2, \ldots, N_{\mathrm{r}},
$$

where $i, j$ are the sequential numbers of the individual batch and run respectively, and $l$ is the accepted length of the MA operation.

\subsection{Run-to-Run optimization}

Run-to-Run (RtoR) optimization is a well-established method in a case of repetitive (batch) processes in chemical and bio-chemical industries [3,28]. In this work the model based on RtoR optimization is accepted because of availability of proved mathematical models $[9,13,15]$. Limiting the model parameters $P=\left\langle P_{1}, P_{2}\right\rangle$ to lie to some defined set $P \in] P[$ the run-to run optimization may be formulate as a static optimization problem where we seek to minimize the accepted objective function $\pi$ :

$$
\underset{P, \xi}{\min i m i z e} \underset{P(P, \lambda)}{\pi} \text { if } P \in] P[\& \xi \in \Lambda .
$$

In (20) $\lambda$ is the index of the accepted alternative structure of the control system and $] P[$ is the set of vector parameters.

\subsection{Case-based reasoning}

Case-Based Reasoning (CBR) has been studied in detail in the last two decades $[1,2,23]$. Now it is accepted as a tried and tested approach to implement in a broad variety of practical problems: biological cleaning of waste water [4], color matching [6], different tasks in the information technologies [23], complex systems analysis and control [16, 17, 23], medicine [23], business processes [23].

In the CBR we can use examples of past "problem solution" to solve arising problems. The cases $C$ are presented in the established following "problem $P$ solution $S$ " form:

$$
C=C(\Omega, S)
$$


In this work the situation $\mathrm{H}$ is considered as a problem part $\mathrm{P}$ of the case $\mathrm{C}$. The situation is represented via attributes $\Theta_{i}$ and their values $v_{i}$.

As attributes are accepted to be only variable, this could be measured or estimated according to some rules or on the basis of operational heuristic. In the TTP two types of attributes describe the situation:

$$
H=\Omega=\left(\Theta_{1}, \Theta_{2}\right) \text {. }
$$

The attributes $\Theta_{1}$ are defined by the main technological characteristics of the subjected to TTP wood material:

$$
\Theta_{1}=\left(a_{11}, a_{12}, a_{13}, a_{14}, a_{15}\right),
$$

where $a_{11}$ is the specie of the wood material with its main characteristics: $a_{11}=\left\langle a_{111}, a_{112}\right\rangle ; a_{111}=\rho_{\mathrm{b}}$ is the basic density of the wood; $a_{112}-$ the index of the type of the wood microstructure; $a_{12}=u^{\mathrm{o}}$ - the accepted initial wood moisture content; $a_{13}=T^{\mathrm{0}}$ - the average initial mass temperature of the wood; $a_{14}$ - the index of the geometrical form of the processed wood (round, rectangular); $a_{15}$ - the characteristic dimension of the wood.

The attributes $\Theta_{2}$ are mainly business and technology specified:

$$
\Theta_{2}=\left(a_{21}, a_{22}, a_{23}, a_{24}, a_{25}\right) \text {, }
$$

where $a_{21}=L$ is the relative load of the equipment for TTP (autoclave, pit); $a_{22}=J$ is the accepted objective function

$$
J=\left(J_{1}\left(J_{11}, J_{12}\right), J_{2}, J_{3}\left(J_{1}, J_{2}\right)\right),
$$

$a_{23}$ are the respective technological constraints:

$a_{24}$ are the operational constraints:

$$
a_{23}=\left(G^{\max }, T_{\mathrm{m}}^{\max }\right),
$$

$$
a_{24}=\left(\Gamma_{1}, \Gamma_{2}, \Gamma_{3}, \Gamma_{4}\right),
$$

$a_{25}$ are the admissible terminal thresholds:

$$
a_{25}=\delta \bar{T}\left(\tau_{\mathrm{p}}\right), \delta \bar{v}\left(\tau_{\mathrm{p}}\right) .
$$

The solution part of the case in this work is presented in the following form:

$$
S=\langle E, V, R, W\rangle
$$

where $E$ are the available control structures:

$$
E=\left(l_{1}, l_{2}, l_{3}, l_{4}, l_{5}, l_{6}, l_{7}\right) \text {, }
$$

$V$ is the matrix of parameters of the respective control structure:

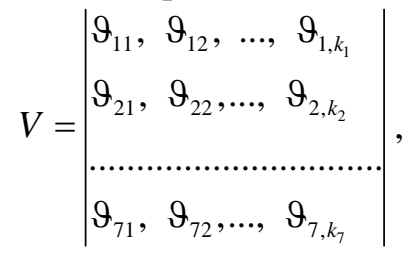

$R$ are the accepted initial settings for the basic temperature control:

$$
R=\left(T_{\mathrm{m}}, \tau_{\mathrm{p}}\right),
$$

$W$ is the final result of the wood thermal treatment of the wood material:

$$
W=\left(v\left(\tau_{\mathrm{p}}\right), \mu\left(\tau_{\mathrm{p}}\right), Q\left(\tau_{\mathrm{p}}\right), \pi\left(\tau_{\mathrm{p}}\right)\right),
$$


where $\pi\left(\tau_{\mathrm{p}}\right)$ is the productivity of the current run.

The well-established $R_{\mathrm{e}}^{4} \mathrm{CBR}$ cycle $[1,2,23]$ modified for the TTP is presented on Fig. 7. The principle of "local-global" closeness $[1,2]$ is adopted by applying similarity measure in the form:

$$
\operatorname{sim}\left(\Omega_{n}, \Omega\right)=\sum_{i=1}^{r} \omega_{i} \operatorname{sim}_{i}\left(a_{n}, a_{i}\right),
$$

where $\Omega_{n}$ and $a_{n}$ are a new problem and its attributes; $\operatorname{sim}\left(a_{n}, a_{i}\right)$ characterizes the local system similarity between the attributes $a_{n}$ and $a_{i}$ and contains mainly specific knowledge of a given local area. The weights $\omega_{i}$ represent the relative importance of these attributes on the common closeness between $\Omega_{n}$ and $\Omega$.

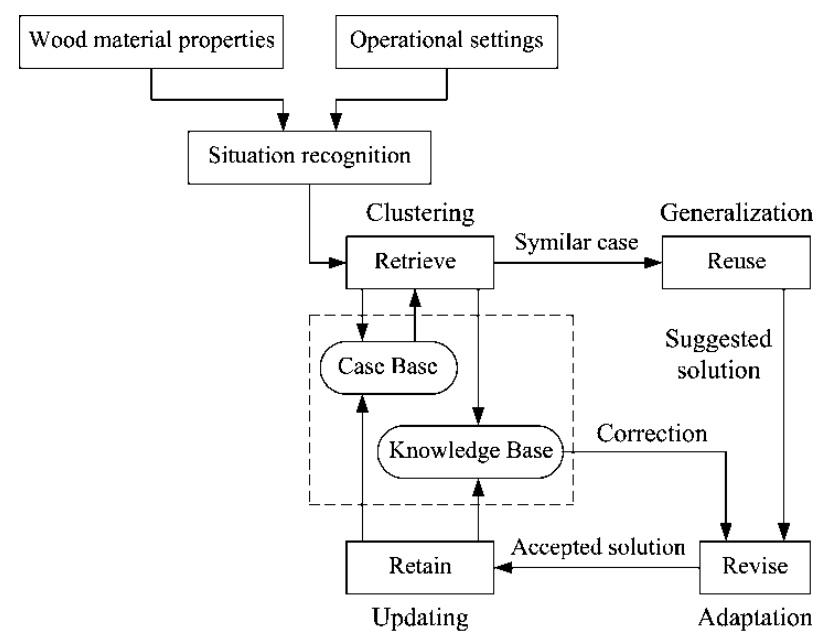

Fig. 7. Scheme of the $R_{\mathrm{e}}^{4}-\mathrm{CBR}$

In TTP considerations both direct measurements of the wood color at the end of the run or ist operator's subjective estimates could be used in CBR. But industrial application could have only the first approach.

\subsection{Control structure formation}

Direct measurement of the relative degree of the wood coloring $v_{\mathrm{DM}}$ gives a variety of possibilities for TTP improvements mainly by:

- Receiving more objective information about the final wood color.

The value of color estimation could be represented in the next optional form:

$$
\hat{v}_{\mathrm{e}}=\alpha_{1} v_{\mathrm{DM}}+\alpha_{2} v_{\mathrm{CBR}}+\alpha_{3} v_{\mathrm{MM}} \text { if } \sum_{i=1}^{3} \alpha_{i}=1 \text {, }
$$

where $v_{\mathrm{DM}}, v_{\mathrm{CBR}}$, and $v_{\mathrm{MM}}$ are respectively the results of the color estimation of the wood material using Direct Measurement (DM), by CBR, and by mathematical model $\left(\mathrm{MM}_{\mathrm{v}}\right)$. The next five combinations for the Control System Structures (CSS) are available (Table 1). 
Table 1. Combinations of the control system structures

\begin{tabular}{|c|c|c|c|c|c|}
\hline CSS & DM & CBR & MM $v$ & MMT & RM \\
\hline $1=$ CSS 1 & 1 & 0 & 0 & - & $v$ \\
\hline $2=$ CSS 2 & 1 & 0 & 0 & $v$ & $v$ \\
\hline $3=$ CSS3 & $\alpha$ & $1-\alpha$ & 0 & $v$ & $v$ \\
\hline $4=$ CSS 4 & $\alpha$ & 0 & $1-\alpha$ & $v$ & $v$ \\
\hline $5=$ CSS5 & $\alpha_{1}$ & $\alpha_{2}$ & $1-\left(\alpha_{1}+\alpha_{2}\right)$ & $v$ & $v$ \\
\hline
\end{tabular}

By using CBR the standard deviation of $\hat{v}_{\mathrm{e}}$ will be reduced especially in cases with large uncertainties.

- Correction of the mathematical models RMi, MMT, and MMv (see Fig. 6).

Because the main space state of the thermal treatment process are immeasurable, some mathematical models have been developed, as follows:

- a set of static regression models [13, 14, 15];

- dynamic models of the type of [18] for averaged $T(x, y, z, k)$ and $v(x, y, z, k)$.

Both groups of models $\left(\mathrm{RM}_{i}, \mathrm{MM}_{\mathrm{T}}\right.$, and $\left.\mathrm{MMv}\right)$ are adapted using Run-to-Run iterative procedure $[3,28]$.

- Control system structure enhancing.

A number of new possible control structures become available after adding of the estimation of the wood color $\hat{v}$ in accordance with Table 1:

- open loop control with adaptation of the mathematical models RMs (CSS1);

- cascaded closed loop control using $\bar{T}$ as a feedback (CSS2);

- cascaded closed loop control using $\bar{T}$ as a feedback with adding CBR (CSS3);

- double cascaded control using both $\bar{T}$ and $v_{\mathrm{e}}$ as feedbacks (CSS4);

- double cascaded control system using both $\bar{T}$ and $v_{\mathrm{e}}$ as feedbacks with CBR (CSS5).

All listed structures in Table 1 with direct measurement of the wood color assure better control performance in comparison with the systems without color estimation in cases when the terminal constraint to achieve the setting color is important. These structures attenuate the impact of uncertainties formulated as difference between averaged and individual values of the parametric vector $P$ in (17).

\section{Peculiarities of the wood ennoblement process control}

\subsection{Flowchart of the process control}

The flowchart of the proposed control algorithm is shown on Fig. 8.

After the situation $\mathrm{H}$ recognition (17) suited cluster for CBR or directly regression model RM is retrieved. Then the operational changes (if any) and current re-optimization are carried out up to the end of the $j$-run, considered in detail in [14]. New information for the wood color achieved into the executed run is fulfilled by direct measurement. Equivalent color estimation $\hat{v}_{\mathrm{e}}$ according (33) and Table 1 is derived and Run-to-Run correction (optimization is addressed toward mathematical models RMs, $\mathrm{MM}_{\mathrm{T}}, \mathrm{MM} \mu, \mathrm{MM} v$, and supervisory level (see Fig. 6). 


\subsection{Problems with the ennoblement process control}

In contrast to TTP addressed mainly to assure a given level of wood plasticity $[9,13,14]$, in the case of ennoblement of the wood material with additional goal to reach desired color some complications arise:

- The plant is TISO king, because $\mu$ and $\nu$ could be changed only by one controlled variable - the temperature of the heating medium Tm through distributed wood material temperature $T(x, y, z)$ and respectively average mass temperature $\bar{T}$ (Fig. 8). Thus kinds of override control have been accepted [17, 18, 19].

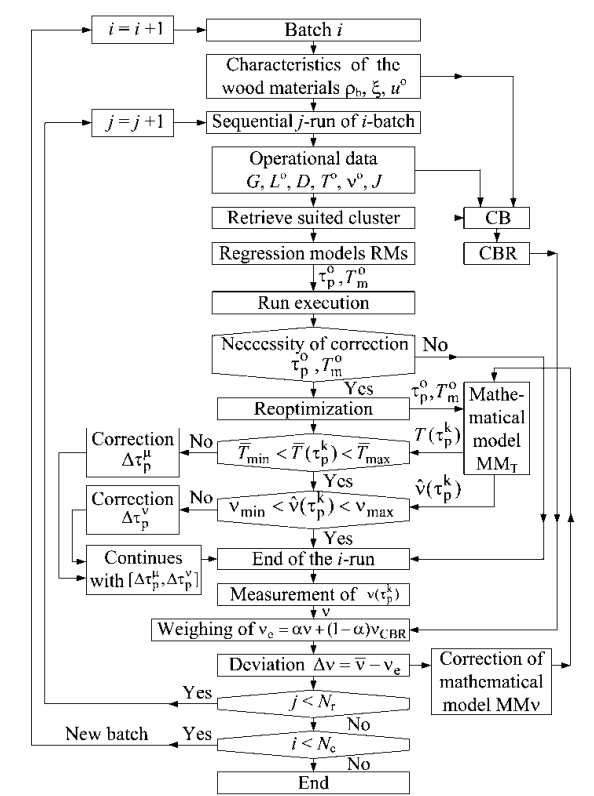

Fig. 8. Flowchart of the control algorithm of the wood ennoblement process

- The technological thresholds for control $\mu$ and $v$ are quite different. The permissible diapason $\Delta \bar{T}$ of the average mass temperature $\bar{T}$ is quite large up to $25-30 \%$ from the setting values (Fig. 9), because could be compensated during the next mechanical processing of the heated wood material.

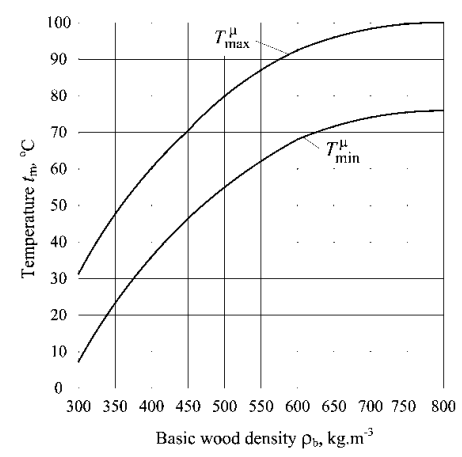

Fig. 9. The domain of optimal wood temperature for thermo mechanical processing 
At the same time the terminal constraint for the wood color given by (16), is very hard because of the producer's requirements and impossibility of any correction as the coloring process is irreversible. Thus the main attention must be paid to the coloring process control.

- The speed of the processes of plasticizing and coloring is quite different (see Fig. 4). The coloring degree strongly depends on the heating medium temperature (Fig. 10). Thus the main control settings $T_{\mathrm{m}}^{\mathrm{o}}$ and $\tau_{\mathrm{p}}^{\mathrm{o}}$ (see Fig. 6) should be carefully coordinated because of possible static contradictions (Fig. 11).

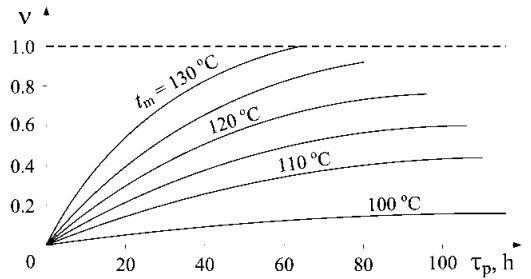

Fig. 10. Relative colorization, depending on the duration of TTP

\subsection{The influence of uncertainty}

On Fig. 12 a real situation is presented, where the discrepancy $\Delta v$ between the degree of coloring $v_{\mathrm{MM}}$ estimated from the mathematical model $\mathrm{MM} v$ and directly measured $v_{\mathrm{DM}}$ is significant. This means that the terminal constraint $v\left(\tau_{\mathrm{p}}\right)$ will be very far from the setting value $v^{0}$ and the run production should be rejected (if $v_{\mathrm{DM}}\left(\tau_{\mathrm{p}}\right)>v^{0}$ ) with additional losses of energy. Thus all accepted models $\mathrm{RMs}, \mathrm{MM}_{\mathrm{T}}, \mathrm{MM} \mu$, and $\mathrm{MM} v$ must be adapted Run-to-Run.

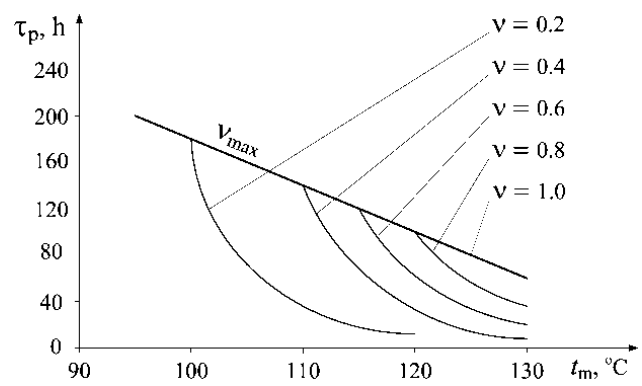

Fig. 11. $\left(t_{\mathrm{m}}-\tau_{\mathrm{p}}\right)$-constraints in the wood coloring by TTP

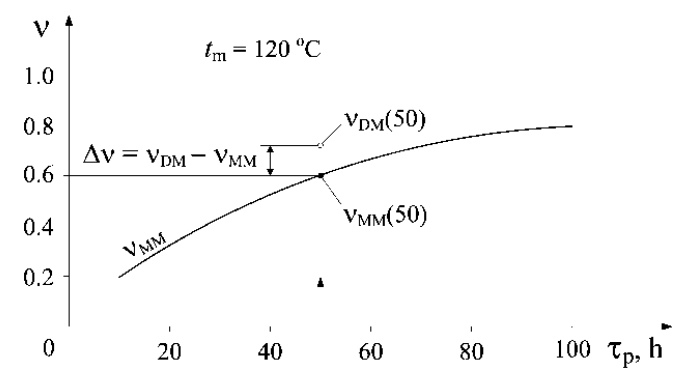

Fig. 12. Difference $\Delta v$ between measured ( $\left.v_{\mathrm{DM}}\right)$ and predicted ( $\left.v_{\mathrm{MM}}\right)$ values of the wood coloring 
Following the results, received in our previous works [13, 14, 15], a suboptimal TTP control could be presented as a choosing of the working point A belonging to the curve 1 (Fig. 13) satisfying the equation:

$$
F\left(T_{\mathrm{m}}^{*}, \tau_{\mathrm{p}}^{*}, \bar{P}_{i}, v^{\mathrm{o}}\right)=0,
$$

where $T_{\mathrm{m}}^{*}$ and $\tau_{\mathrm{p}}^{*}$ are the suboptimal values of the control cycle, $\bar{P}_{i}$ is the averaged parameter in $i$-batch, and $v^{\circ}$ is the setting value of the desired degree of wood coloring. As unknown vector of the parameters in $(i, j)$-run

$$
P_{i, j} \neq \bar{P}_{i},
$$

the corresponding curve will be different and some corrections $A \rightarrow B$ or $A \rightarrow C$ will be needed as shown on Fig. 13.

When a multi-criteria approach (9) is accepted, a re-optimization is accomplished $A \rightarrow F$ (Fig. 14), where the additional objective function is minimizing of the heat consumption $Q$ (curves 3 and 4).

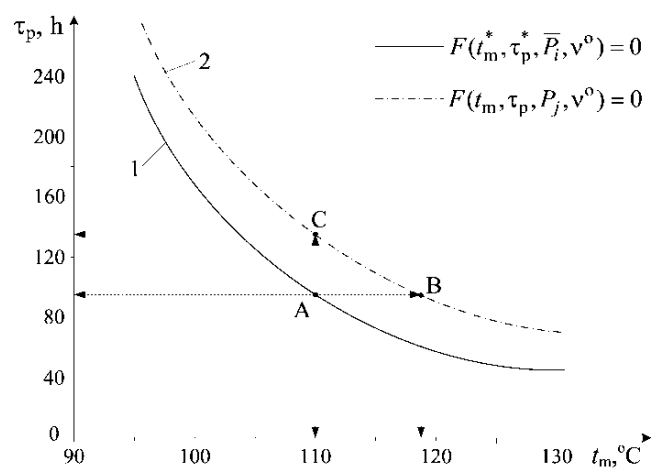

Fig. 13. Necessary corrections of the control cycle settings of $t_{\mathrm{m}}$ and $\tau_{\mathrm{p}}$

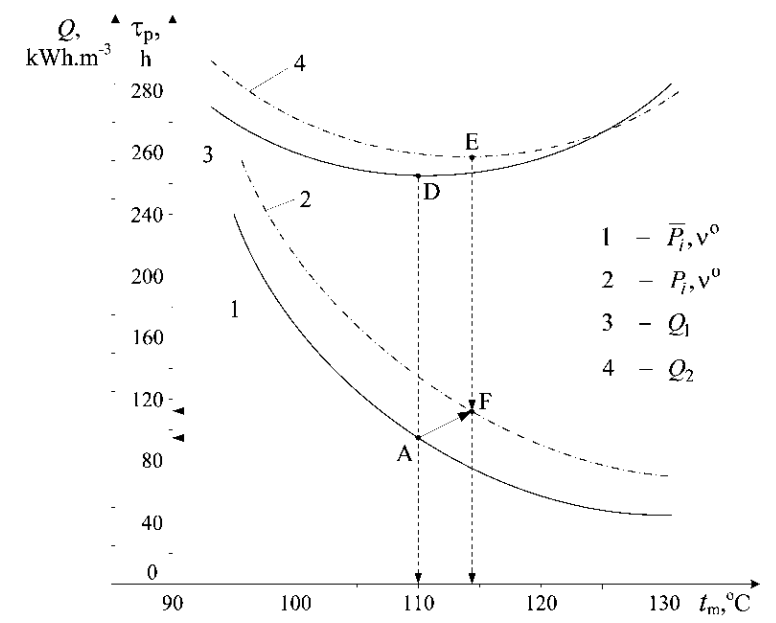

Fig. 14. Re-optimization of the control cycle settings $T_{\mathrm{m}}^{*}$ and $\tau_{\mathrm{p}}^{*}$ by Run-to-Run procedure 


\section{Experimental results}

\subsection{The color measurement}

The color of the beech wood details before and after their autoclave steaming was measured using CIE- $\mathrm{L}^{*}, \mathrm{a}^{*}, \mathrm{~b}^{*}$ color space coordinates as a components to estimate the degree of coloring $v$. The obtained results from the experiments before and after three steaming regimes are presented in Table 2 and Table 3.

Table 2. Main data from the experimental measurement of the wood color

\begin{tabular}{|l|c|c|c|c|c|}
\hline \multirow{2}{*}{ Parameters } & \multirow{2}{*}{$\begin{array}{c}\text { Before } \\
\text { steaming }\end{array}$} & \multicolumn{3}{c|}{ After steaming } \\
\cline { 4 - 6 } & & Regime 1 & Regime 1 & Regime 3 \\
\hline \multirow{2}{*}{$\begin{array}{l}\text { Temperature of the processing } \\
\text { medium, }{ }^{\circ} \mathrm{C}\end{array}$} & $t_{\mathrm{m}}^{\min }$ & 20 & 110 & 125 & 135 \\
\cline { 4 - 6 } & $t_{\mathrm{m}}^{\max }$ & 20 & 115 & 130 & 140 \\
\hline \multicolumn{2}{|l|}{ Number of measurements } & 166 & 57 & 54 & 55 \\
\hline \multicolumn{2}{|l|}{ Duration of the steaming process, h } & - & 7.5 & 8.5 & 9.5 \\
\hline
\end{tabular}

The change in the total color deviation $\Delta E^{*}$ of beech details in dependence on the steaming regime is shown on Fig. 15.

Table 3. Change in the CIE L*a*b* color coordinates, depending on the steaming regime

\begin{tabular}{|l|c|c|c|c|c|c|}
\hline & \multicolumn{2}{|c|}{$\mathrm{L}^{*}$} & \multicolumn{3}{c|}{$\mathrm{a}^{*}$} & \multicolumn{3}{c|}{$\mathrm{b}^{*}$} \\
\cline { 2 - 7 } & value & $\sigma, \%$ & value & $\sigma, \%$ & value & $\sigma, \%$ \\
\hline Before steaming & 73.5 & 2.8 & 7.6 & 36.8 & 19.8 & 9.1 \\
\hline Steaming regime 1 & 68.4 & 4.1 & 10.1 & 22.8 & 20.6 & 6.3 \\
\hline Steaming regime 2 & 58.1 & 6.5 & 15.5 & 14.2 & 24.9 & 5.2 \\
\hline Steaming regime 3 & 53.2 & 5.1 & 17.9 & 11.2 & 26.1 & 5.7 \\
\hline
\end{tabular}

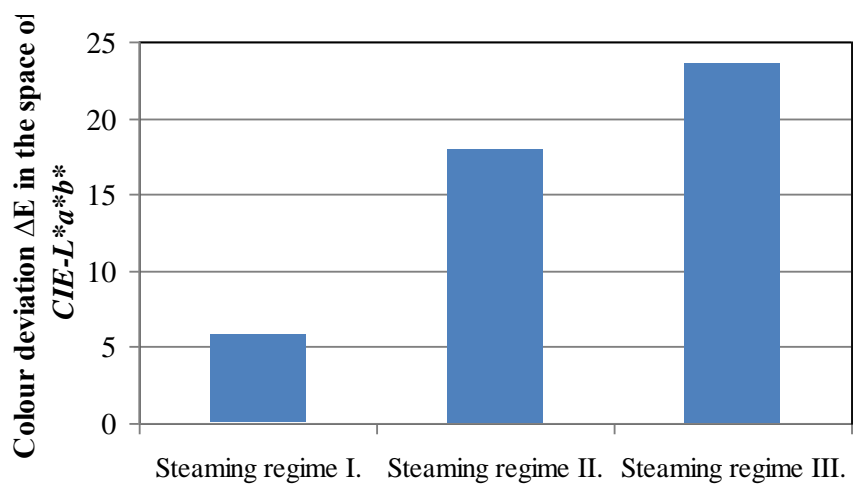

Fig. 15. Change in the total color deviation $\Delta E^{*}$ of beech details before and after their steaming, depending on the steaming regime

The experiments show that the accepted method based on CIE-L*, a*, b* standard (ISO 11664-4:2008) using a colorimeter Colour Reader CR-10 (Konica Minolta, Japan) gives representative estimates of the wood material coloring and could be used in Run-to-Run optimization. 


\subsection{Practical realization}

Different variants of the proposed control system (see Fig. 6) have been implemented in more than 20 Small and Medium Enterprises (SMEs) in Bulgaria and Slovakia [9, 13]. Industrial PLC and PID controllers have been used in the basic feedback control (Fig. 16). The results show 2-3 time reduction of the specific (for $1 \mathrm{~m}^{3}$ wood) energy consumption as well as a strong unification of the quality of the processed wood material.
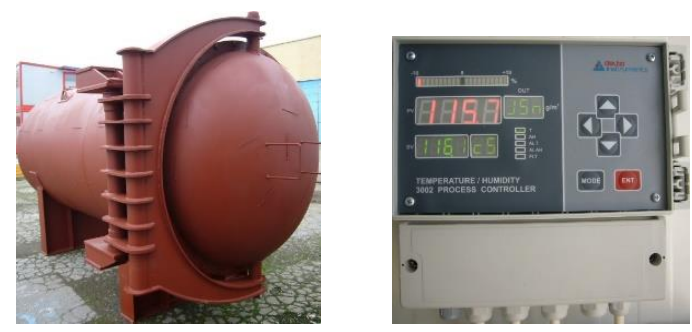

Fig. 16. Autoclave (left) and PLC in a system for model-based control of the wood steaming process in it (right)

\section{Conclusion}

An enhancement of the functionality of the existing control systems for the thermal treatment of wood materials is considered in this paper, especially for the cases with wood coloring as a desired result of this treatment. A direct measurement of the wood color is accepted to be incorporated in the system. This reduces the sensitivity of the control system performance on the existing large uncertainties due to the wood material properties variations and operational changes.

In the control system design an iterative approach is accepted with optional including of different functional building blocks. Special attention is paid to the representation and utilization in a relevant way of the existing operational knowledge in form of Case Based Reasoning. Some of the proposed alternative control structures have been realized in the industrial practice with clear cost reduction.

\section{References}

1. A a m o d t, A., E. P 1 a s a. Case-Based Reasoning: Fondatational Issues, Methodological Variations and System Approach. - AI Communications, Vol. 7, 1994, No 1.

2. B e r g m a n, R., M. R i c h t e r, S. S h m i t t, A. S t a h l, I. W o 11 r a t o. Utility Oriented Matching: A New Direction for Case-Based Reasoning. - In: Proc. of 1st Conf. on Professional Knowledge Management, Shaker, 2001.

3. B o n vi n, D., B. S r i n i v a s a n, D. R u p p e n. Dynamic Optimization in the Batch Chemical Industry. - In: AIChE Symposium Series, 2002, pp. 255-279.

4. B o s h n a k o v, K., M. H a d j i s k i, Z. G e or g i e v. Control of Process in Biological Cleaning of Waste Water Using Case-Based Reasoning (Part 1 and Part 2). - In: Proc. of Int. Conf. on Automatics and Informatics, 2013, Sofia.

5. B r o s i l o w, C., B. J o s e p h. Technologies of Model Based Control. New Jersey, Prentice Hall, 2002.

6. C h e e t h a m, W, J. G r a f. Case-Based Reasoning in Colour Matching. - In: Proc. of 2nd Int. Conf. on CBR (ICCBR'97), Providence, RI, Springer, 1997. 
7. D e 1 i i s k i, N. Transient Heat Conduction in Capillary Porous Bodies. - In: A. Ahsan, Ed., Convection and Conduction Heat Transfer. - In: Rieka, Tech Publishing House, 2011, pp. 149-176. ISBN 978-953-307-582-2. http:// dx.doi.org/10.5772/21424

8. D e l i i s k i, N. Modelling of the Energy Needed for Heating of Capillary Porous Bodies in Frozen and Non-Frozen States. Saarbrücken, Germany, Scholars' Press, 2013. 116 p. ISBN 978-3-639-70036-7. http://www.scholars-press.com//system/ covergenerator/build/1060

9. D e $1 \mathrm{i}$ i s k i, N., L. D z u r e n d a. Modelling of the Thermal Treatment in the Technologies for Wood Processing. Technical University in Zvolen, Slovakia, 2010. 224 p. ISBN 978-954-323-719-7 (in Russian).

10. D o y 1 e, F. J. Nonlinear Inferential Control for Process Applications. - In: J. Process Control, Vol. 8, 1998, No 5-6.

11. D z u r e n d a, L., N. D e 1 i i s k i. Mathematical Model for Evaluation of Heat Energy Norm at Color Homogenization of Beech Lumber in Pressure Autoclaves. - In: Proc. of 6th Int. Conf. "Chip and Chipless Woodworking Processes", Sturovo, Slovakia, 2008, pp. 307-314.

12. D z u r e $\mathrm{n} \mathrm{d} \mathrm{a,} \mathrm{L.,} \mathrm{N.} \mathrm{D} \mathrm{e} 1$ i i s k i. Change of the Colour of Steamed Beech Wood in the Colour Space of CIE L*a*b*. Woodworking Industry, Moscow, 2013, No 3, pp.18-23.

13. H a d j i s k i, M., N. D e 1 i i s k i. Model Based Control of Thermal Treatment of Wood Materials. - In: Proc. of 23rd Int. Conf. "Control of Power Plants and Systems", Bankya, Bulgaria, 2015, pp. 9-13 (in Bulgarian).

14. H a d j i s k i, M., N. D e 1 i i s k i. Re-Optimization of the Wood Thermal Treatment Near Optimal Control. - In: Proc. of Int. Conf. on "Automatics \& Informatics", 2015, Sofia.

15. H a d j i s k i, M., N. D e l i i s k i. Cost Oriented Suboptimal Control of the Thermal Treatment of Wood Materials. - In: Proc. of 16th IFAC Conference on Technology, Culture, and International Stability, 2015, Sozopol, Bulgaria. IFAC-PapersOnLine 45-24. DOI: 10.1016/j.ifacol.2015.12.056. www.sciencedirect.com

16. H a d j i s k i, M., V. B o i s h i n a. Hybrid Supervisory Control of Complex Systems Incorporating Case-Based Reasoning. - In: Proc. of Int. Conf. on Complex Systems, COSY, 2011, Ohrid, Macedonia.

17. H a d j i s k i, M., V. B o i s h i n a. Enhancing Functionality of Complex Plant Hybrid Control Systems Using Case-Based Reasoning (CBR). - In: Proc. of 5th IEEE Int. Conf. on Intelligent Systems, IS'2010, London, UK, 2010.

18. $\mathrm{H}$ a d j i s k i, M. Hybrid Modelling and Inference Model-Based Control of Complicated Technological Plants. - Studies in Informatics and Control, Vol. 8, 1999, No 1.

19. H a d j i s k i, M., K. B o s h n a k o v, N. C h r i s t o v a. Coordinated Inference Control of Interconnected Multistage Processes. - In: Proc. of ASI’99, Leuven, Belgium, 1999.

20. J. Colibri 2014. http://gain.fdi.ucm.es./rerearch/colibri/jcolibri

21. K 1 e m e n t, I., P. M a r k o. Colour Changes of Beech Wood During High Temperature Drying Process. - In: Wood Research, Vol. 54, 2009, No 3, Bratislava.

22. M o r e n o, R., F. D e v l i e g e r. Influence of Wood Characteristics and of Heat Conditioning Parameters on Heating Time of Logs. - In: Ciencia e Investigation Forestal, 1990, No 4.

23. H. Munoz-Avila, F. Ricci, Eds. Case-Based Reasoning: Research and Development, Springer, 2005.

24. S h u b i n, G. S. Drying and Thermal Treatment of Wood. Lesnaya Promyshlennost, 1990, Moscow (in Russian).

25. V i d e 1 o v, H. Drying and Thermal Treatment of Wood. University of Forestry, 2003, Sofia (in Bulgarian).

26. V i g a k, V. M. Optimal Control of Non-Stationary Temperature Regimes. Kiev, Naukova Dumka, 1979 (in Russian).

27. W a t s o n, I. Applying Case-Based Reasoning: Techniques for Enterprise Systems. Morgan Kaufmann, SF, 1977.

28. W e 1 z, C., B. S r i n i v a s a n, D. B o n v i n. Combined On-Line and Run-to-Run Optimization of Batch Processes with Terminal Constraints. - In: Proc. of ADCHEM'03, 2004.

29. Z h o u, J., C. W e n. Adaptive Backstopping Control of Uncertain Systems. Springer, LNCIS, 2015. 